\title{
Selective Laser Trabeculoplasty Versus MIGS: Forgotten Art or First-Step Procedure in Selected Patients with Open-Angle Glaucoma
}

\author{
Milena Pahlitzsch • Anja-Maria Davids · Sibylle Winterhalter • \\ Malte Zorn · Emanuel Reitemeyer • Matthias K. J. Klamann • \\ Necip Torun · Eckart Bertelmann · Anna-Karina Maier
}

Received: March 17, 2021 / Accepted: April 24, 2021 / Published online: May 7, 2021

(C) The Author(s) 2021

\section{ABSTRACT}

Introduction: To evaluate the long-term effect on intraocular pressure (IOP) and glaucoma medication of selective laser trabeculoplasty (SLT) compared to minimally invasive glaucoma surgery (MIGS) in primary open-angle glaucoma (POAG) and its potential in clinical practice.

Methods: A total of 342 consecutive patients (stand-alone procedures) were included. One hundred and five patients underwent SLT treatment $\left(360^{\circ}\right.$ SLT, $95-105$ spots, Trabeculas SLT ARCLaser, Nürnberg, DE), 107 patients had an ab interno-derived trabeculotomy (Trabectome $^{\circledR}$, NeoMedix, Tustin, USA), and 130

Milena Pahlitzsch and Anja-Maria Davids shared first author.

Meeting: 2018 DOC German Ophthalmology Society, Nürnberg, Germany.

Supplementary Information The online version contains supplementary material available at https:// doi.org/10.1007/s40123-021-00347-0.

M. Pahlitzsch ( $\square)$ - A.-M. Davids · S. Winterhalter .

M. Zorn · E. Reitemeyer · M. K. J. Klamann ·

N. Torun · E. Bertelmann - A.-K. Maier

Department of Ophthalmology, Charité -

Universitätsmedizin Berlin, Corporate Member of Freie Universität Berlin, Berlin Institute of Health, Humboldt-Universität Zu Berlin, Augustenburger Platz 1, 13353 Berlin, Germany

e-mail: milena.pahlitzsch@charite.de patients received iStent inject ${ }^{\circledR}$ implantation (2 implants-Glaukos, CA, USA). IOP and glaucoma therapy were evaluated preoperatively, 1 day, 6 weeks, 3 months, 6 months, and 1, 2, and 3 years postoperatively. Statistical analysis was performed using a regression model and propensity matching score (reduced cohort number) using SPSS v20.0. Kaplan-Meier analysis was included using the following six criteria: criterion $\mathrm{A} \quad(\mathrm{IOP} \leq 21 \mathrm{mmHg}$ with or without medication, qualified success), criterion $\mathrm{B}$ (IOP $\leq 18 \mathrm{mmHg}$ with or without medication, qualified success), criterion C (IOP $\leq 21 \mathrm{mmHg}$ without medication, complete success), criterion D (IOP $\leq 18 \mathrm{mmHg}$ without medication, complete success), criterion $\mathrm{E} \quad(\mathrm{IOP} \leq 21 \mathrm{mmHg}$ and IOP reduction $>20 \%$ after therapy), and criterion $\mathrm{F}$ (IOP $\leq 18 \mathrm{mmHg}$ and IOP reduction $>20 \%$ after therapy).

Results: In the matched cohort, the SLT cohort showed an IOP reduction of $31.2 \%$ from $19.9 \pm 2.3$ to $13.7 \pm 2.7 \mathrm{mmHg} \quad(p<0.001)$ 3 years postoperatively; in Trabectome ${ }^{\circledR}$ IOP decreased by $31.4 \%$ from $20.5 \pm 1.3$ to $13.8 \pm 2.0 \mathrm{mmHg}(p<0.001)$ and in iStent inject $^{\circledR}$ by $29.9 \%$ from $19.5 \pm 2.0$ to $13.8 \pm 2.7 \mathrm{mmHg} \quad(p<0.001) . \quad$ Trabectome $^{\circledR}$ and iStent inject ${ }^{\circledR}$ could not demonstrate a significant reduction in glaucoma therapy (Trabectome $^{\circledR} \quad p=0.138, \quad$ iStent inject $^{\circledR}$ $p=0.612$ ); a significant drop was noted in SLT $(2.2 \pm 1.2$ to $1.7 \pm 1.2, p=0.046)$. SLT and 
MIGS achieved good to moderate survival rates using criterion A $(93.3 \%$ SLT, $79.7 \%$ Trabectome $^{\circledR}, 77.6 \%$ iStent inject ${ }^{\circledR}$ ) and criterion B (74.5\% SLT, $48.0 \%$ Trabectome ${ }^{\circledR}, 56.2 \%$ iStent inject $\left.{ }^{\circledR}\right)$. As expected, low survival rates were obtained with non-filtering procedures: criterion C $11.1 \%$ in SLT, $6.5 \%$ in Trabectome ${ }^{\circledR}$, $7.0 \%$ in iStent inject ${ }^{\circledR}$ and criterion D 3.0\% in SLT, $4.3 \%$ in Trabectome $^{\circledR}, 3.7 \%$ in iStent inject ${ }^{\circledR}$ in 3-year follow-up.

Conclusion: The SLT is a low-complication and effective method for reducing pressure in mild to moderate POAG. SLT is suitable as an initial procedure when setting up a step scheme; MIGS is the treatment of choice as a follow-up for mild to moderate forms of glaucoma and accepted topical therapy. Ethic approval had been given by the Ethikkommission Charite Universitätsmedizin Berlin, EA4/047/20—retrospectively registered.

Keywords: Selective laser trabeculoplasty (SLT); Minimally invasive glaucoma surgery (MIGS); Primary open-angle glaucoma (POAG); iStent inject $^{\circledR}$; Trabectome $^{\circledR}$

\section{Key Summary Points}

Why carry out this study?

Unmet need of non-invasive treatment in patients with glaucoma without longterm damage or allergic reaction of glaucoma medication.

Established selective laser therapy (SLT) might delay glaucoma progression by significantly reducing the intraocular pressure.

SLT might present an alternative to minimal invasive glaucoma surgery (MIGS) and should not be considered a forgotten art.

\section{What was learned from the study?}

Selective laser therapy showed an IOP reduction of $31.2 \%$ from $19.9 \pm 2.3$ to $13.7 \pm 2.7 \mathrm{mmHg}(p<0.001) 3$ years postoperatively.
SLT can be stated as an effective intervention without a complication profile in a treatment plan for mild to moderate glaucomatous damage over a period of 3 years.

MIGS showed-as expected-a significant IOP reduction in mild to moderate POAG.

SLT can be stated as an alternative method to stabilize IOP levels in POAG.

\section{DIGITAL FEATURES}

This article is published with digital features, including a summary slide, to facilitate understanding of the article. To view digital features for this article go to https://doi.org/10.6084/ m9.figshare.14473434".

\section{INTRODUCTION}

For several years, the field of minimally invasive glaucoma surgery (MIGS) has been evolving with numerous devices and procedures having already been introduced to the market [1-8]. Most publications analyzing these new minimally invasive surgical approaches present promising results which are reflected by a significant intraocular pressure (IOP) reduction and low complication rate [1-8].

From the very beginning, Trabectome ${ }^{\circledR}$ and iStent inject ${ }^{\circledR}$ have been at the forefront of the MIGS field. This raises the question of whether these surgical interventions differ from each other regarding their outcome and are able to sustain a comparison with conventional procedures-such as the selective laser trabeculoplasty (SLT)—over a 3-year period after surgery.

All three procedures address the angle of the anterior chamber in different ways. On the one hand, Trabectome ${ }^{\circledR}$ and iStent inject ${ }^{\circledR}$ are examples of a Schlemm's canal-based surgery. This can be understood in that the Trabectome ${ }^{\circledR}$ opens and the iStent inject ${ }^{\circledR}$ bypasses the trabecular meshwork as a major aqueous outflow barrier in order to lower the IOP which is the 
major risk factor for the development and progression of glaucoma [5-8]. The iStent inject ${ }^{\circledR}$ received US Food and Drug Administration (FDA) approval in 2012. During the surgery, two heparin-coated, non-ferromagnetic titanium microstents with a snorkel shape are implanted into the trabecular meshwork and thereby bypass it [5-8]. The Trabectome ${ }^{\circledR}$, on the other hand, was introduced in 2004 and consists of a one-use, disposable handpiece that is used for electrocautery, irrigation, and aspiration $[6,7]$. It works by removing an arc of $90-120^{\circ}$ of trabecular meshwork and the inner wall of Schlemm's canal, which leads to a facilitated drainage of aqueous humor $[6,7]$.

SLT, on the other hand, is a trabeculoplasty performed with the doubled pulsed-frequency of neodymium (Nd):YAG laser which selectively targets pigmented tissue of the trabecular meshwork $[9,10]$. Although mechanical, cellular, and biochemical theories of action are discussed, the exact mechanism of the selective laser trabeculoplasty is still poorly understood even today $[9,10]$.

Ultimately, it is the aim of this study to assess whether there are significant differences in the long-term outcome (3 years worth of data), the safety of the Trabectome ${ }^{\circledR}$ and iStent inject ${ }^{\circledR}$ procedure, and to compare the results with SLT.

\section{METHODS}

The selected, retrospective, single-center study design is congruent with the ethical principles and criteria for medical research as outlined in the most recent Declaration of Helsinki and took place between October 2014 and December 2018. Ethics approval had been given by the Ethikkommission, Charité - Universitätsmedizin Berlin, EA4/047/20. For this type of study formal consent is not required. An informed written consent was provided for surgery and laser treatment.

For this study, a total of 342 patients with primary open-angle glaucoma (POAG) were included, who underwent one of the two surgeries (Trabectome $^{\circledR}$ or iStent inject ${ }^{\circledR}$ standalone) or the laser procedure (SLT) between
October 2014 and December 2018 at the Charité.

All patients demonstrated glaucomatous optic disc alterations and an open chamber angle in the gonioscopy (Shaffer III-IV, wellpigmented trabecular meshwork). The optic disc was examined using the diagnostic criteria described by Jonas, depending on the degree of glaucomatous cupping [11]. Additionally, the retinal nerve fiber layer (RNFL) thickness in all quadrants and the cup-disc ratio (CDR) was measured preoperatively to add information to the glaucoma severity status (optic coherence tomography, Spectralis-OCT, Heidelberg, Germany). The inclusion criteria entailed best-corrected visual acuity of at least 20/200 (6/60), reliable visual field testing (three successive visual fields with reproducible visual field defects due to glaucomatous damage), and informed patient consent for surgery. In measuring the IOP, the well-known Goldmann applanation tonometry standard was used [12]. No washout phase of topical glaucoma medication was established in this retrospective cohort study.

There were grounds of exclusion in the consideration of some patients, such as those who presented themselves with active inflammation in the anterior as well as posterior chamber, or those with corneal infections. Additional criteria for exclusion were also considered in patients presenting higher spherical errors ( $>5$ diopters), higher astigmatism (> 2.5 diopters), hazy optic media interfering with fundus examination, and ocular trauma. Patients who underwent intraocular surgery less than 3 months before the study were also excluded. Lastly, patients who used contact lenses within the last 3 months were excluded from the study. However, prior history of contact lenses was not considered for grounds of exclusion.

In all subsequent appointments following the procedures IOP readings, visual acuity and the number of glaucoma medications were evaluated. Patients attended consultations preoperatively, and follow-ups 1 day, 6 weeks, 3 months, 6 months, 1 year, 2 years, and 3 years post surgery. 
The surgical technique was chosen by the surgeon, following the patient's individual needs in terms of glaucoma therapy and IOPlowering effect based on the factors recommended by the European Glaucoma Society including the stage of glaucoma, IOP before treatment, age and life expectancy, rate of progression, and presence of risk factors for progression. The progression rate was defined by progredient visual field defects, IOP elevation above the individual limit (please see European Glaucoma Society for IOP reduction in different glaucoma levels) and worsening in the various diagnostic tools (confocal laser scanning tomography, optical coherence tomography, and fundus camera). Surgical treatments were performed using the same protocols under local anesthesia.

\section{Selective Laser Trabeculoplasty (SLT)}

By way of definition, the SLT is a procedure using laser treatment for glaucoma therapy. This procedure was executed in the following way for this study: the $360^{\circ}$ SLT treatment was performed with Trabeculas SLT (A.R.C. Laser, Nuernberg, Germany) using 90-100 spots applied to the trabecular meshwork [13-15].

\section{Trabectome ${ }^{\circledR}$ Surgery (Stand-Alone Procedure)}

In brief: a 1.8-mm near limbal temporal corneal incision was made, an ophthalmic viscosurgical solution was injected into the anterior chamber for structural stability, and the Trabectome ${ }^{\circledR}$ (NeoMedix, Inc., Tustin, CA, USA) handpiece was inserted. Under gonioscopic view, the selective electrosurgical ablation was activated to remove an approximately $120^{\circ}$ arc of trabecular meshwork and an inner wall of the Schlemm canal. The surrounding tissue and collector channels were shielded from the electric energy with a ceramic-coated footplate [7, 16-19].

\section{iStent Inject ${ }^{\circledR}$ Implantation (Stand-Alone Procedure)}

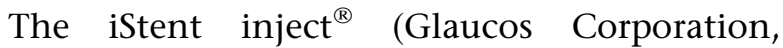
Laguna Hills, CA, USA) sole procedure started with a 1.8-mm self-sealing, temporal, clear corneal incision. Acetylcholine chloride 1\% (Miochol) was only injected into the anterior chamber of eyes with still narrow pupils after a topical pilocarpine treatment preoperatively. Afterwards, an ophthalmic viscosurgical device was injected into the anterior chamber to improve structural stability. Under gonioscopic view, the injector was then advanced nasally and two iStents were implanted through the trabecular meshwork into Schlemm's canal, separated by approximately 2 clock hours $[5,8,20]$.

\section{Statistics}

Statistical data was calculated using Statistical Package for the Social Sciences (SPSS) version 20.0, from SPSS, Inc.; descriptive statistics (mean, standard deviation, 95\% limits of agreement, and correlation quotients) were processed. A power analysis based on the effect sizes noted in prior research indicated that a sample population of 109 individuals (our study $=342$ ) would be required to detect a correlation coefficient of 0.3 with an $90 \%$ power and an $\alpha=0.05$. Normality was tested for all outcome measures. For normally distributed data, differences were assessed by unpaired Student's $t$ test (two groups) and one-way analysis of variance (ANOVA; three groups) for continuous variables. For non-normal distribution, the Mann-Whitney $U$ test and Kruskal-Wallis $H$ test were performed respectively. Post hoc comparisons were done using the Bonferroni test. Student's $t$ test for paired samples and Wilcoxon sign ranked test was performed in case of normal or non-normal distribution of paired data, respectively. Differences in categorical data were assessed using the chi-squared test.

A multiple regression analysis was carried out with IOP at year 3 as the outcome variable. Baseline IOP, baseline medication, global RNFL 
thickness, CDR, and type of procedure were predictors of the model.

The propensity score for matching was estimated using a logistic regression model with the study data, in which the type of procedure was regressed on baseline IOP and glaucoma medication. The calculated propensity scores were then used for 1:1 ratio matching of the groups using nearest matching approach within a caliper distance of 0.2 of the standard deviation of the logit of the propensity score.

Kaplan-Meier analysis was conducted using the following six criteria: criterion A (IOP $\leq 21 \mathrm{mmHg}$ with or without medication, qualified success), criterion B (IOP $\leq 18 \mathrm{mmHg}$ with or without medication, qualified success), criterion $\mathrm{C}$ (IOP $\leq 21 \mathrm{mmHg}$ without medication, complete success), criterion D (IOP $\leq 18 \mathrm{mmHg}$ without medication, complete success), criterion $\mathrm{E}$ (IOP $\leq 21 \mathrm{mmHg}$ and IOP reduction $>20 \%$ after therapy) and criterion $\mathrm{F} \quad(\mathrm{IOP} \leq 18 \mathrm{mmHg}$ and $\mathrm{IOP}$ reduction $>20 \%$ after therapy). A $p$ value of less than 0.05 indicated a statistically significant difference.

\section{RESULTS}

Data from 342 patients with open-angle glaucoma were analyzed in three study cohorts (SLT $n=105$, iStent inject ${ }^{\circledR} n=130$, Trabectome ${ }^{\circledR}$ $n=107)$. The patients in the SLT group were significantly younger than both MIGS groups and there were significantly more female patients in the SLT group compared to both MIGS groups (Tables 1, 2).

In the univariable model, IOP at year 3 was not significantly associated with baseline medication, CDR, global RNFL, and type of procedure. Nevertheless, we found differences in baseline IOP $(p=0.003)$. In the multivariable model adjusted by potentially confounding variables, the IOP at year 3 was still significantly associated with baseline IOP $(p<0.001)$, while no relationship was found with the type of procedure $(p=0.094)$ (Table 3 ).

Using the propensity score matching, we obtained the following data (Table 2): 66 patients undergoing a Trabectome ${ }^{\circledR}$ surgery (66 eyes, mean age $71.5 \pm 9.6$ years), iStent inject ${ }^{\circledR}$ procedures (66 eyes, mean age $76.0 \pm 8.9$ years), and selective laser trabeculoplasties (66 eyes, mean age $65.5 \pm 13.3$ years).

In the matched collective, IOP was reduced from preoperative values $19.9 \pm 2.3 \mathrm{mmHg}$ in SLT, $20.5 \pm 1.3 \mathrm{mmHg}$ in Trabectome ${ }^{\circledR}$, and $19.5 \pm 2.0 \mathrm{mmHg}$ in iStent inject $^{\circledR}$ to $13.7 \pm 2.7 \mathrm{mmHg} \quad$ (SLT, $\quad p<0.001$ ), $13.8 \pm 2.0 \mathrm{mmHg} \quad$ (Trabectome $^{\circledR}, \quad p<0.001$ ), and $13.8 \pm 2.7 \mathrm{mmHg}$ (iStent ${ }^{\circledR}, p<0.001$ ) at 3 years postoperative follow-up. Mean IOP reduction was $31.2 \%$ in SLT, $31.4 \%$ in Trabec-

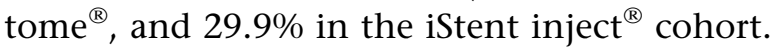

No significant IOP difference was found between SLT compared to MIGS ( $p=0.987)$. IOP data is illustrated in Fig. 1.

Glaucoma medication was reduced from $2.2 \pm 1.2$ preoperatively in SLT, $2.4 \pm 0.7$ in Trabectome $^{\circledR}$, and $2.2 \pm 1.2$ in iStent inject ${ }^{\circledR}$ to $1.7 \pm 1.2$ in SLT $(p=0.046), 1.9 \pm 1.1$ in Trabectome $^{\circledR}$ cohort $(p=0.138)$, and $1.7 \pm 1.5$ in iStent inject ${ }^{\circledR}(p=0.612) 3$ years postoperatively. Mean glaucoma therapy reduction over the follow-up period of 3 years was $22.8 \%$ in the SLT, $21.8 \%$ in the Trabectome ${ }^{\circledR}$, and $22.8 \%$ in the iStent inject ${ }^{\circledR}$ cohort.

No significant difference in glaucoma medication was noted between MIGS techniques and SLT $(p=0.930) 3$ years after surgery. Glaucoma medication over time is plotted in Fig. 2.

Reduction of preoperative medication was found in the Trabectome ${ }^{\circledR}$ cohort. More specifically, reduction of glaucoma medication was observed in $62.5 \%$ of all cases. In $25.0 \%$ of the cohort, one medication was reduced, and in $37.5 \%$, two or more glaucoma medications were decreased.

In the SLT cohort, reduction of medication was found in $25.0 \%$ of all patients, in $15.0 \%$ reduction by one medication, and in $10.0 \%$ by two or more medications.

In the iStent inject ${ }^{\circledR}$ cohort, a reduction of medication was found in $41.7 \%$ of all cases, in $33.3 \%$ by one medication, and in $8.4 \%$ by two or more glaucoma medications.

The Kaplan-Meier analysis revealed the following results (matched cohort, demonstrated in Fig. 3): SLT and MIGS achieved good to moderate survival rates using criterion $\mathrm{A}$ at 
Table 1 Demographic statistics in the complete study cohort

\begin{tabular}{|c|c|c|c|c|}
\hline & $p$ value 3 groups (Kruskal-Wallis) & SLT & iStent inject $^{\circledR}$ & Trabectome $^{\circledR}$ \\
\hline \multicolumn{5}{|l|}{ Age (years) } \\
\hline$N$ & $<0.001$ & 105 & 130 & 107 \\
\hline Mean \pm SD & & $65.2 \pm 11.9$ & $73.4 \pm 9.5$ & $71.2 \pm 10.8$ \\
\hline \multicolumn{5}{|l|}{ Gender (\%) } \\
\hline Male & 0.031 & 29.5 & 45.4 & 36.6 \\
\hline Female & & 70.5 & 54.6 & 66.4 \\
\hline \multicolumn{5}{|l|}{ Eye (\%) } \\
\hline OD & $<0.001$ & 49.5 & 44.2 & 46.7 \\
\hline OS & & 50.5 & 55.8 & 53.3 \\
\hline \multicolumn{5}{|l|}{ IOP $(\mathrm{mmHg})$} \\
\hline$N$ & $<0.001$ & 105 & 129 & 105 \\
\hline Mean \pm SD & & $17.9 \pm 3.5$ & $20.2 \pm 6.1$ & $23.3 \pm 4.8$ \\
\hline \multicolumn{5}{|l|}{ Medication } \\
\hline$N$ & $<0.001$ & 105 & 130 & 90 \\
\hline Mean \pm SD & & $1.7 \pm 1.20$ & $2.3 \pm 1.1$ & $2.5 \pm 0.9$ \\
\hline \multicolumn{5}{|l|}{ CDR } \\
\hline$N$ & $<0.001$ & 99 & 55 & 33 \\
\hline Mean \pm SD & & $0.6 \pm 0.2$ & $0.8 \pm 0.2$ & $0.6 \pm 0.2$ \\
\hline \multicolumn{5}{|c|}{ RNFL global $(\mu \mathrm{m})$} \\
\hline$N$ & $<0.001$ & 93 & 23 & 31 \\
\hline Mean \pm SD & & $81.9 \pm 16.3$ & $75.1 \pm 20.2$ & $67.8 \pm 19.6$ \\
\hline
\end{tabular}

$S L T$ selective laser trabeculoplasty, IOP intraocular pressure, medication number of glaucoma medications, $R N F L$ retinal nerve fiber layer, $C D R$ cup to disc ratio, mean $\pm S D$

1-year follow-up (93.3\% SLT, $84.1 \%$ Trabectome $^{\circledR}, 77.6 \%$ iStent inject ${ }^{\circledR}$ - qualified success) and at 3-year follow-up (93.3\% SLT, 79.7\% Trabectome $^{\circledR}, 77.6 \%$ iStent inject ${ }^{\circledR}$-qualified success) and criterion B at 1-year follow-up (83.9\% SLT, 56.8\% Trabectome $^{\circledR}, 64.2 \%$ iStent inject ${ }^{\circledR}$-qualified success) and at 3-year followup $\left(74.5 \%\right.$ SLT, $48.0 \%$ Trabectome $^{\circledR}, 56.2 \%$ iStent inject ${ }^{\circledR}$-qualified success).

As expected of non-filtering procedures, criterion C (complete success) and criterion D (complete success) showed low survival rates (Fig. S1 in the supplementary material): criterion C $11.1 \%$ in SLT, $49.1 \%$ in Trabectome $^{\circledR}, 38.7 \%$ in iStent inject ${ }^{\circledR}$ and criterion D $8.9 \%$ in SLT, $32.8 \%$ in Trabectome ${ }^{\circledR}, 29.6 \%$ in iStent inject $^{\circledR}$ in 1-year follow-up and criterion C $11.1 \%$ in SLT, $6.5 \%$ in Trabectome ${ }^{\circledR}$, $7.0 \%$ in iStent inject ${ }^{\circledR}$ and criterion D $3.0 \%$ in SLT, $4.3 \%$ in Trabectome ${ }^{\circledR}, 3.7 \%$ in iStent inject $^{\circledR}$ in 3-year follow-up. In addition, criterion $\mathrm{E} \quad(\mathrm{IOP} \leq 21 \mathrm{mmHg}+\mathrm{IOP} \quad$ reduction $>20 \%$ after surgery) and criterion $\mathrm{F}$ (IOP $\leq 18 \mathrm{mmHg}+$ IOP reduction $>20 \%$ after surgery) displayed the following results of the survival rate (Fig. S2 in the supplementary 
Table 2 Demographic statistics in the matched study cohorts

\begin{tabular}{llll}
\hline & SLT & iStent inject $^{\circledR}$ & Trabectome $^{\circledR}$ \\
\hline$N$ (eyes) & 66 & 66 & 66 \\
Gender (male/female) & $45.4 \% / 54.6 \%$ & $46.7 \% / 53.3 \%$ & $48.8 \% / 51.2 \%$ \\
Age (years) & $65.5 \pm 13.3$ & $76.0 \pm 8.9$ & $71.5 \pm 9.6$ \\
Visual acuity (decimal) & $0.74 \pm 0.22$ & $0.64 \pm 0.29$ & $0.63 \pm 0.26$ \\
Degree of anterior chamber angle & Shaffer III-IV & Shaffer III-IV & Shaffer III-IV \\
IOP (mmHg) baseline & $19.9 \pm 2.3$ & $19.5 \pm 2.0$ & $20.5 \pm 1.3$ \\
Medication & $2.2 \pm 1.2$ & $2.2 \pm 1.2$ & $2.4 \pm 0.7$ \\
Cup to disc ratio (mean) & $0.52 \pm 0.1$ & $0.71 \pm 0.1$ & $0.68 \pm 0.1$ \\
RNFL global $(\mu \mathrm{m})$ & $81.8 \pm 16.2$ & $71.5 \pm 21.8$ & $68.7 \pm 19.6$ \\
\hline
\end{tabular}

$S L T$ selective laser trabeculoplasty, IOP intraocular pressure, medication number of glaucoma medications, $R N F L$ retinal nerve fiber layer, mean $\pm \mathrm{SD}$

Table 3 Results of the univariable and multivariable regression analysis on the intraocular pressure (IOP) at year 3

\begin{tabular}{|c|c|c|c|c|}
\hline \multirow[t]{2}{*}{ Variable } & \multicolumn{2}{|l|}{ Univariable model } & \multicolumn{2}{|l|}{ Multivariable model } \\
\hline & Coefficient (95\% CI) & $p$ value & Coefficient (95\% CI) & $p$ value \\
\hline Baseline IOP ( $\mathrm{mmHg}$ ) & $0.171(0.058-0.283)$ & 0.003 & $0.552(0.386 / 0.718)$ & $<0.001$ \\
\hline Baseline medication & $-0.320(-0.862$ to 0.222$)$ & 0.244 & $-0.716(-1.411 /-0.022)$ & 0.044 \\
\hline CDR & $-3.008(-6.839$ to 0.823$)$ & 0.122 & $1.740(-2.750 / 6.230)$ & 0.439 \\
\hline Global RNFL & $0.037(-0.017$ to 0.091$)$ & 0.176 & $0.03(-0.022 / 0.074)$ & 0.275 \\
\hline Surgery type & $0.536(-0.277$ to 1.348$)$ & 0.194 & $1.14(-0.203 / 2.485)$ & 0.094 \\
\hline
\end{tabular}

$R N F L$ retinal nerve fiber layer, $C D R$ cup to disc ratio

material): criterion E $53.1 \%$ in SLT, $27.2 \%$ in Trabectome $^{\circledR}, 30.2 \%$ in iStent inject ${ }^{\circledR}$ and criterion F $55.4 \%$ in SLT, $27.2 \%$ in Trabectome ${ }^{\circledR}$, $30.2 \%$ in iStent inject ${ }^{\circledR}$ in 1 year follow-up and criterion E $53.1 \%$ in SLT, $13.6 \%$ in Trabectome $^{\circledR}, 30.2 \%$ in iStent inject ${ }^{\circledR}$ and criterion $\mathrm{F}$ $46.0 \%$ in SLT, $13.6 \%$ in Trabectome ${ }^{\circledR}, 30.2 \%$ in iStent inject ${ }^{\circledR}$ in 3-year follow-up.

Follow-up interventions (trabeculectomy, cyclophotocoagulation, Re-MIGS) were necessary for uncontrolled, individual IOP in $4.5 \%$ of the iStent inject ${ }^{\circledR}$ cohort and $18.1 \%$ of the Trabectome $^{\circledR}$ group over a period of time (see Table 4 for details); $1.5 \%$ of SLT-treated patients needed further glaucoma surgery in the followup period of 3 years (required MIGS).
The most common complication was intraoperative blood reflux, which occurred in almost all eyes out of the surgically treated eyes and none of the SLT cohort. Furthermore, there were no incidences noted of sustained hypotony, choroidal effusion or hemorrhage, visual acuity decrease, infection, aqueous misdirection, or wound leakage.

\section{DISCUSSION}

The appeal of MIGS is based on easy implementation, low complication rate, and efficiency in lowering the IOP. With Trabectome ${ }^{\circledR}$ and iStent inject ${ }^{\circledR}$, an adequate IOP-lowering 


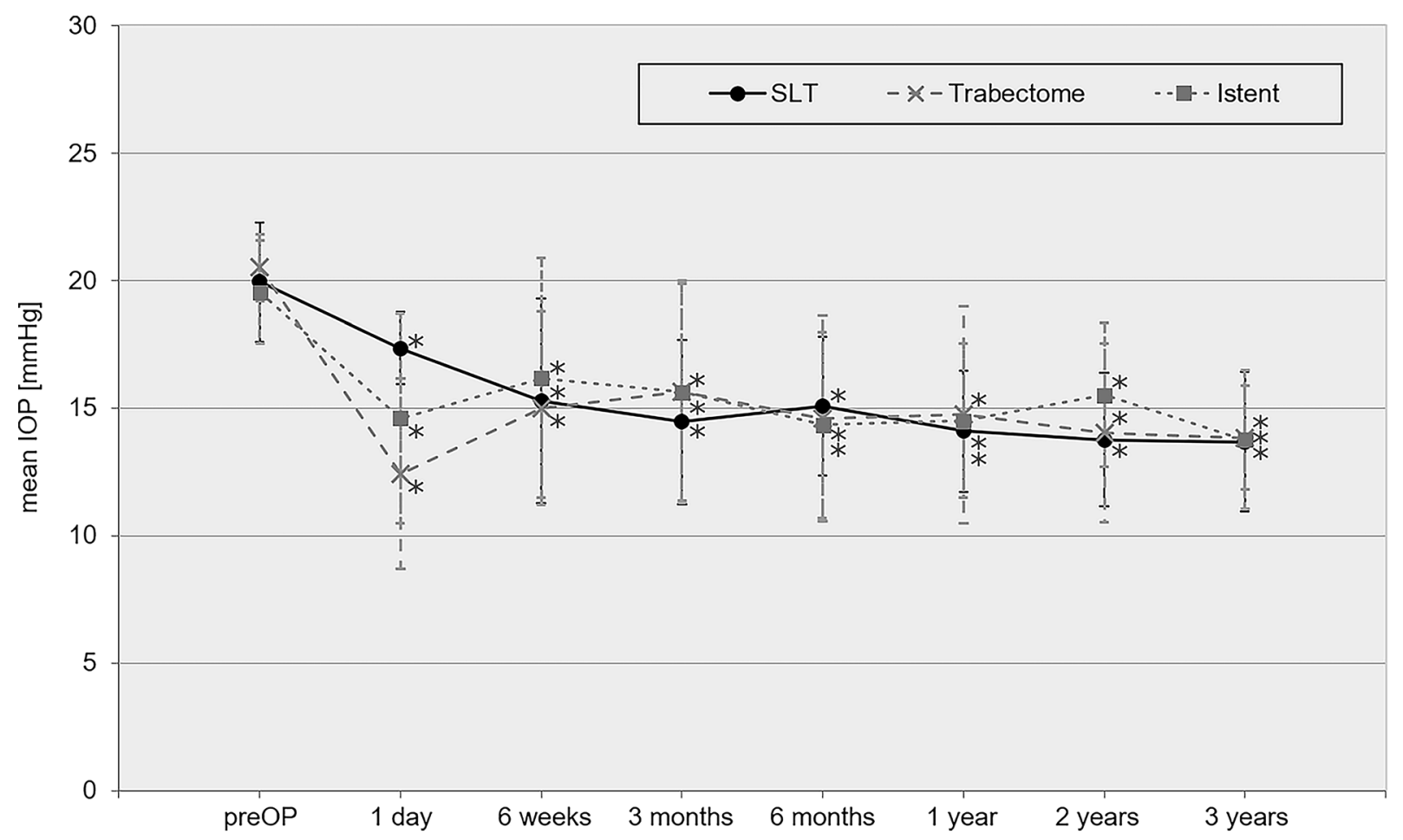

Fig. 1 Trendline chart of the intraocular pressure comparing preoperative to postoperative follow-up data in the matched cohort

effect has already been observed in multiple studies in at least the 12 months' postoperative period $[8,16-18,20]$.

In addressing the subsequent question of how this effect evolves 3 years after the surgery, it is furthermore necessary to take a step back and compare the outcome of these new approaches with proven established procedures. As "magic dwells in each beginning", new procedures might detract from the effectiveness and safety of conventional methods like SLT, for example, which then become neglected.

As one should always select a treatment that is individually customized to the patient's requirements and general condition, and longterm outcomes of MIGS, such as iStent inject ${ }^{\circledR}$ and Trabectome ${ }^{\circledR}$, should be compared with each other and with conventional procedures like SLT.

\section{No Significant Difference Between SLT, Trabectome $^{\circledR}$, and iStent Inject ${ }^{\circledR}$ in Lowering the IOP}

In the case of iStent inject ${ }^{\circledR}$, the average IOP reduction is reported to be $26.2-46.9 \%$ at $12-$ 18 months $[8,21,22], 34.5 \%$ at 24 months, and $26.2 \%$ at 3 years [23]. Donnenfeld et al. published a success rate of $89.7 \%$ after 3 years (defined as IOP $\leq 18 \mathrm{mmHg}$ ) [23]. As they washed out the glaucoma medication before performing the iStent ${ }^{\circledR}$ implantation, their results are comparable with our outcome only to a limited extent. Additionally, in randomized clinical trials using iStent inject ${ }^{\circledR}$ as the sole procedure, the average IOP reduction seems to be higher and was $31.3-38.4 \%$ at $12-18$ months $[19,24]$, $45.9 \%$ at 24 months, and $42.8 \%$ at 3 years [25]. However, Vold et al. only included newly diagnosed POAG and randomized the patients to two trabecular bypass stents or prostaglandin eye drops [25]. Thus, this study is not ideal for a comparison with our data as we also included 


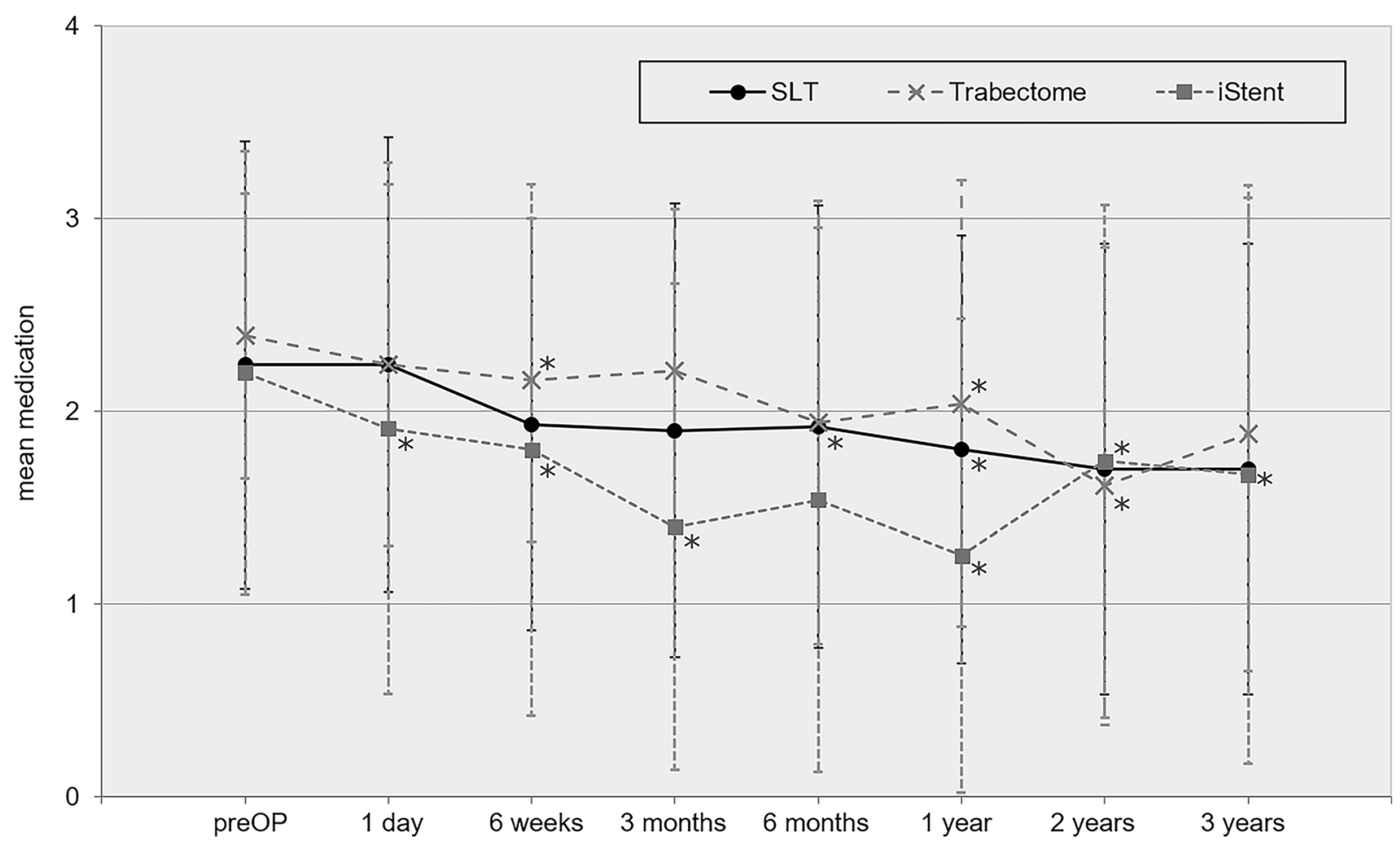

Fig. 2 Trendline chart of the number of glaucoma medications comparing preoperative to postoperative follow-up data in the matched cohort
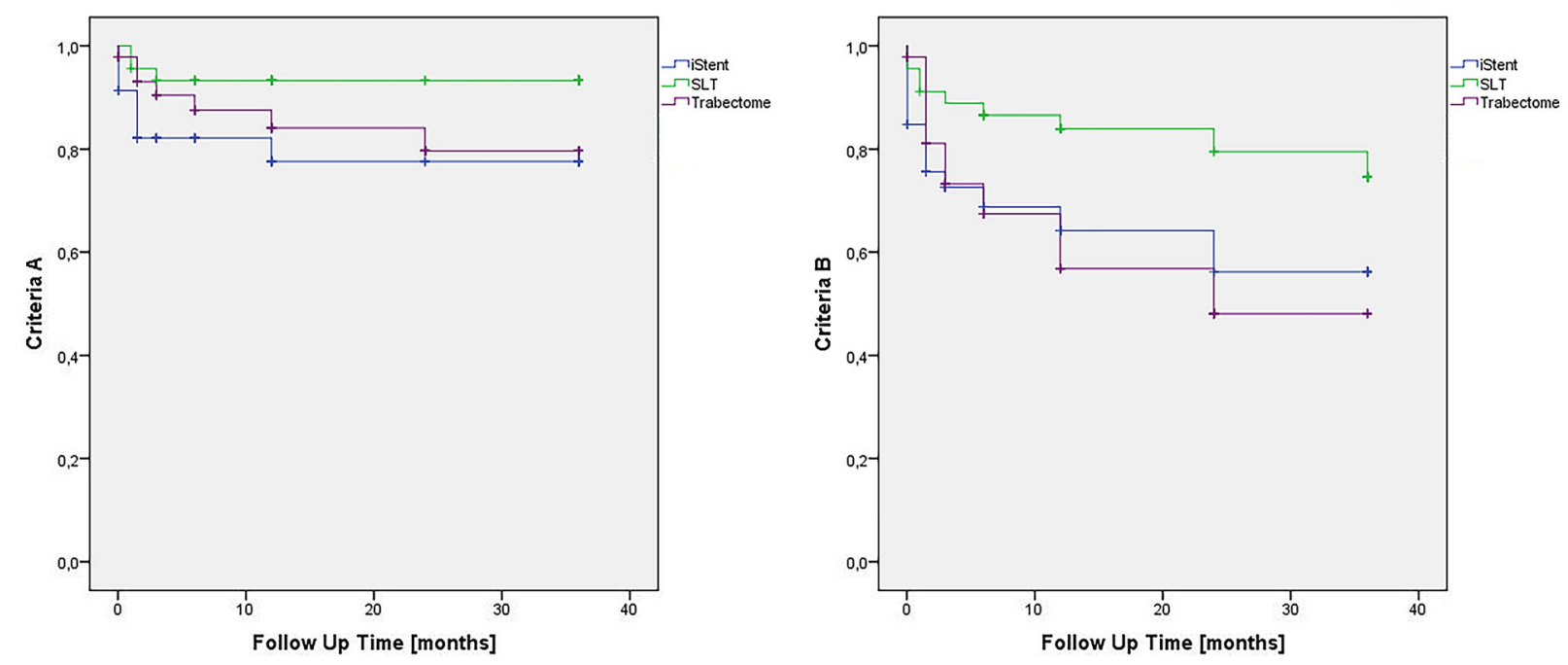

Fig. 3 Kaplan-Meier analysis of the matched cohort including the following criteria: criterion A (IOP $<21 \mathrm{mmHg}$ with or without medication, qualified success) and criterion B (IOP $<18 \mathrm{mmHg}$ with or without medication, qualified success)

patients with a known POAG diagnosis and history of different topical medication. Another reason for the divergent IOP reduction might be that in these randomized clinical trials, the daily clinical routine is not perfectly reflected.

In our study cohort, we documented an IOP reduction of $29.9 \% 3$ years after the surgery 


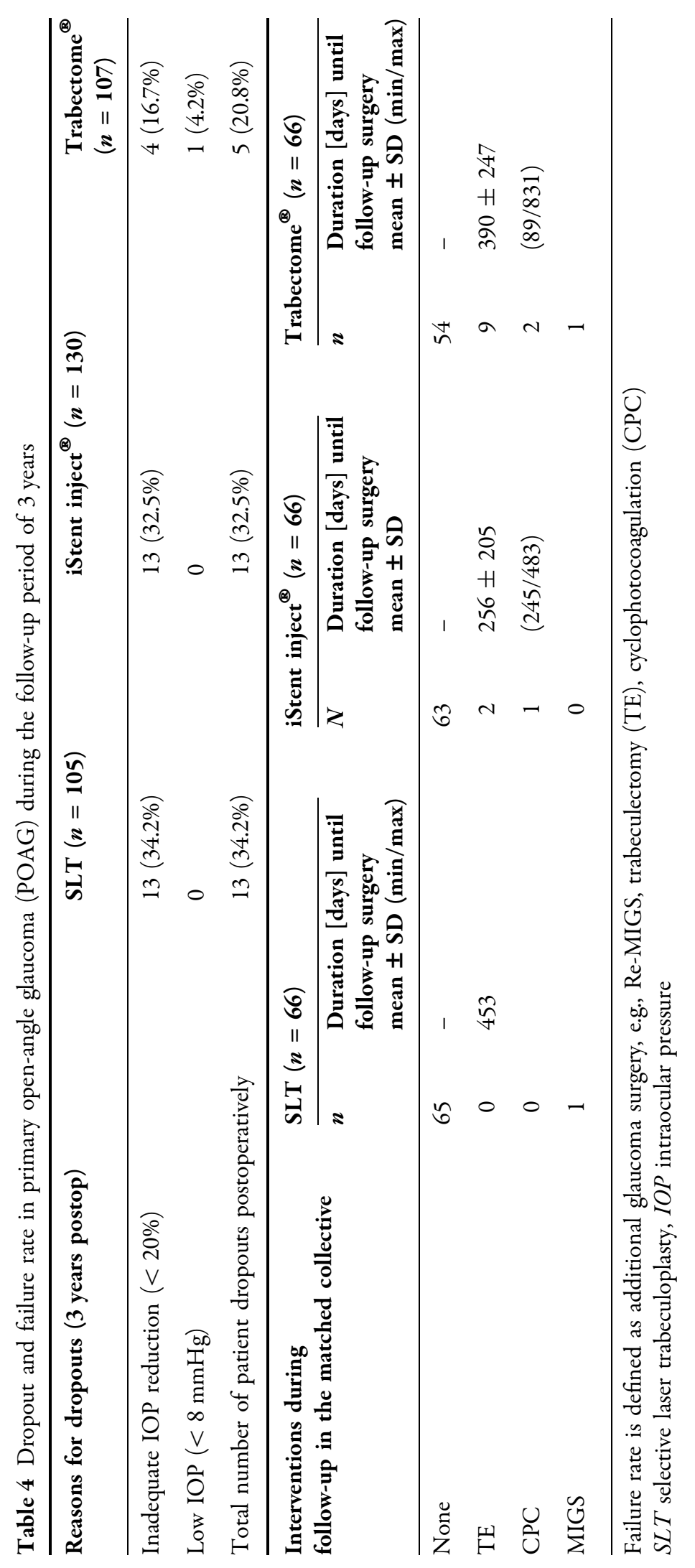


with a survival rate of $77.6 \%$ (criterion A; defined as IOP $<21 \mathrm{mmHg}$ ) and of $56.2 \%$ (criterion $\mathrm{B}$; defined as IOP $<18 \mathrm{mmHg}$ ), respectively. Criterion E (30.2\% in iStent inject $\left.{ }^{\circledR}\right)$ and criterion $\mathrm{F}\left(30.2 \%\right.$ in iStent inject $\left.{ }^{\circledR}\right)$ showed a drop of the survival rates. These results indicate that MIGS needs to be carefully chosen to constantly lower the IOP in patients with glaucoma. In summary, the average IOP-lowering effect we analyzed in our 3-year data is midrange compared with published IOP-reduction data.

Regarding the Trabectome ${ }^{\circledR}$-only cases, publications about non-randomized prospective and retrospective studies reveal an IOP reduction of $24.9-34.1 \%$ at 12 months [17, 26-28], $40 \%$ at 24 months [20], $41 \%$ at $30-36$ months $[6,29], 40 \%$ at 48 months, and $32 \%$ at 60 months $(n=2)$ [6]. Minckler et al., who had the largest cohort of Trabectome ${ }^{\circledR}$-only cases ( $n=738, n=35$ after 36 months; $72.6 \%$ POAG), reported a success rate of approximately $65 \%$ (defined as IOP $\leq 21 \mathrm{mmHg}$ and IOP reduction $\geq 20 \%$ from baseline IOP) [6]. In our study, we documented an IOP reduction of $31.4 \% 3$ years after the surgery with respective survival rates of $79.7 \%$ (criterion A; defined as IOP $<21 \mathrm{mmHg}$ ) and $48.0 \%$ (criterion $\mathrm{B}$; defined as IOP $<18 \mathrm{mmHg}$ ). Applying even stricter criteria for analyzing survival rates (criteria $\mathrm{C}-\mathrm{F}$ ), we find the expected drop of the success rate with criteria E + F $\left(13.6 \%\right.$ in Trabectome $\left.{ }^{\circledR}\right)$ (see Figs. S1 and S2 in the supplementary material). If these criteria reflected the real-life outcome of our patients, we would recommend MIGS in a different way [30]. Although most of the study patients could not achieve an IOP reduction $>20 \%$ without medication, we do see an individually controlled IOP in the daily routine and slowing down of the glaucoma progression (matched cohort IOP reduction $>20 \%$ : iStent inject ${ }^{\circledR} 83 \%$, Trabectome ${ }^{\circledR} 87.5 \%$, SLT $\left.94.4 \%\right)$. Hence, our data ranges at the lower level of the published IOP-reduction following Trabectome ${ }^{\circledR}$ surgery.

In non-randomized clinical trials involving SLT, the average IOP reduction following the laser treatment is documented to be $13-21 \%$ at 24 months [31-33] and $24.1-29.3 \%$ at 48 60 months [34-36]. Especially regarding the long-term data, randomized clinical trials show a similar IOP reduction with $16.9-26.4 \%$ at 12 months [37, 38], $7.7 \%$ at 24 months [39], and $32.1-33 \%$ at 60 months $[40,41]$.

In their review, Leahy and White summarize that on the basis of the commonly adopted success criteria of IOP reduction $>20 \%$ from baseline IOP, success rates vary from $66.7 \%$ to $75 \%$ at 6 months, $58-94 \%$ at 12 months, $40-85 \%$ at 2 years, $38-74 \%$ at 3 years, $38-68 \%$ at 4 years, and $11.1-31 \%$ at 5 years. Consequently, they concluded that the IOP-lowering effect of SLT diminishes over time [42].

In accordance with the published data, we documented in our study cohort an IOP reduction of $31.2 \% 3$ years after the surgery with good respective survival rates of $53.1 \%$ (criterion $\mathrm{E}$ ) and $46.0 \%$ (criterion $\mathrm{F}$ ). In contrast to the result concluded in Leahy and White, we could not detect a diminishing effect at least over the period of 3 years.

So far, no other publication can be found comparing the outcome of MIGS (iStent inject ${ }^{\circledR}$, Trabectome $^{\circledR}$ ) and SLT. Only three original publications (to the best of our knowledge) exist which compare iStent inject ${ }^{\circledR}$ implantation with the Trabectome ${ }^{\circledR}$ surgery. These publications have a follow-up of only 12 months and combine the respective procedure with cataract extraction, i.e., not analyzed as stand-alone procedures. Two of these studies did not show a significant difference between both groups $[43,44]$, whereas one study reported a significant greater IOP reduction and less glaucoma medication in the iStent ${ }^{\circledR}$ subgroup [45].

Additionally, one paper was found comparing SLT with iStent inject ${ }^{\circledR}$ regarding their costs [46]. Berdahl et al. showed in their analysis that despite higher costs in year 0 , annual costs thereafter were lowest in the two-stent treatment arm and showed savings compared to the medications only and SLT treatment arms [46].

In our study we found no significant differences in IOP-lowering effect between the three matched groups and also in the multiple regression analysis of the complete cohort. Additionally, postoperative IOP at year 3 was not significantly associated with the type of procedure. Therefore, it seems that there is no difference in the IOP-lowering effect between 
all three interventions. This is an interesting aspect as there were assumptions made that the laser treatment would lead to a weaker IOP reduction than invasive glaucoma surgery. It has to be taken into account that there is a selection bias in the glaucoma severity and this could be a reason for the missing difference, but the IOP-lowering effect in the matched groups was comparable. At this time we aim to evaluate these retrospective data to develop an easy decision-making process for the treatment of mild to moderate POAG in a clinical routine setting. In general, prospective studies need to be conducted to avoid the preoperative bias.

\section{Exclusively Selective Laser \\ Trabeculoplasty Significantly Lowers the Number of Glaucoma Medications}

As a result of different study designs, the number of publications that have comparable results to this study in analyzing the impact of SLT, iStent inject ${ }^{\circledR}$, and Trabectome ${ }^{\circledR}$ on glaucoma medication are limited. Concerning the iStent inject $^{\circledR}$, a reduction in glaucoma medication of $9.8 \%$ after $12-18$ months $[19,24]$ or $1-1.64$ medications after 18 months [21, 22, 24] is reported in comparative literature. Regarding Trabectome ${ }^{\circledR}$ surgery, the number of glaucoma medications was reduced by $0.57-1$ after $12-$ 24 months $[20,26,27]$. As stated in other literature, the effect in reducing the number of glaucoma therapies seems to be greater after iStent inject ${ }^{\circledR}$ surgery, which is not in accordance with our results. In the iStent ${ }^{\circledR}$ subgroup, a reduction of $22.8 \%$ and 0.5 medications after 36 months was documented.

In our Trabectome ${ }^{\circledR}$ subgroup, we observed a reduction in glaucoma therapy of $21.8 \%$. This result is congruent with published data over a longer period of time.

In our matched study cohort, we observed a significant reduction in glaucoma medication only in the SLT subgroup with $22.8 \%$ and 0.5 medications after 36 months. In the case of the SLT subgroup, our outcome does not comply with the published data where a reduction in glaucoma medication of 1.21 after 18 months [47] and 0.7 after 5 years [40] is reported. When considering the statistical significance, the differences between SLT and MIGS need to be interpreted with caution. Target IOP levels for SLT might be higher as patients experience satisfactory results which allows them to more easily reduce glaucoma medication than compared to MIGS.

In conclusion, all three procedures seem to have a reducing impact, which is-at least in our matched study design-only significant in the SLT subgroup.

Patients need to be informed about the maximum possible effect of medication reduction and must receive clarification about the limits of MIGS and laser treatment prior to surgery. This ambivalence must be taken into consideration in case of drug intolerance as an indication for surgery.

\section{Low Follow-Up Interventions in the SLT and iStent Inject ${ }^{\circledR}$ Cohort}

Follow-up interventions (trabeculectomy, cyclophotocoagulation, Re-MIGS) were necessary for uncontrolled individual IOP in $4.5 \%$ of the iStent inject $^{\circledR}$ cohort and $18.1 \%$ of the Trabectome $^{\circledR}$ group over the period of 3 years (Table 4). Our data showed a low failure rate compared with published data defining failure as additional surgical procedures. Minckler et al. performed additional glaucoma surgery in 100 out of 738 Trabectome procedures (14\%) because of uncontrolled target pressure, usually in the first year post surgery [6]. Follow-up procedures of up to $35 \%$ following Trabectome ${ }^{\circledR}$ surgery can be found in comparative data [29]. Risk factors such as lower preoperative IOP and young patients were noted by Jea et al. [29] Thus, our failure rate of the Trabectome ${ }^{\circledR}$ group is in agreement with comparative literature; however, it is found to be the highest of our three study cohorts. This might be due to a pronounced scarring process after Trabectome ${ }^{\circledR}$ surgery.

In the case of iStent inject ${ }^{\circledR}$ as the sole procedure, only Voskanyan et al. reported a failure rate of $4 \%$ [8]. Other study groups reported "no serious postoperative complications" $[21,23,25]$ or no additional surgery $[19,22,24]$. 
This is in line with our low failure rate of $4.5 \%$. Comparative studies were limited by a short follow-up of only 12-18 months [19, 21, 22, 24] and different study designs without a defined failure reporting $[23,25]$.

Only $1.5 \%$ of SLT-treated patients needed further glaucoma surgery (MIGS) in the followup period of 3 years which emphasizes the right selection of patients undergoing laser treatment.

\section{Adverse Events and Complications are Rare in iStent Inject ${ }^{\circledR}$, Trabectome ${ }^{\circledR}$, and SLT Procedures}

In our study cohort, no severe complications were documented intra-, peri-, or postoperatively. The sole and most common complication was intraoperative blood reflux, which occurred during iStent inject ${ }^{\circledR}$ and Trabectome ${ }^{\circledR}$ surgery. However, this blood reflux indicates a successful surgical procedure and is therefore desired. Furthermore, there was no incidence of postoperative hypotony, nor were there any incidences noted of sustained hypotony, choroidal effusion or hemorrhage, visual acuity decrease, infection, aqueous misdirection, or wound leakage.

In the SLT subgroup, indications of any adverse event or complications were void.

As noted in comparative literature, the most frequent adverse event following iStent inject ${ }^{\circledR}$ and Trabectome ${ }^{\circledR}$ surgery as stand-alone procedures are IOP spikes with an occurrence rate of $1.1-10.1 \%[8,19]$ and $20 \%$ [33], respectively. No report of postoperative infection or visual acuity loss $>2$ lines due to glaucoma was found.

Regarding SLT, IOP spikes in up to $28 \%$ [48] of cases were documented; specific eyes demonstrated some degree of inflammation, which is usually transient [49]. In summary, all three alternatives are safe procedures and well tolerated with low complication rates.

There are limitations of the study which need to be addressed. This study is limited by a retrospective study design. Accordingly, this setting allowed us, to a certain extent, to align preoperative values of the three cohorts. Despite the selection bias in the glaucoma severity between the three cohorts, we found no significant differences in the IOP-lowering effect of this real-world data. Since the severity of glaucoma should not have any influence on the IOP-lowering effect of a standardized surgical procedure, our real-life data should be considered by choosing a glaucoma procedure in the future. Further prospective studies and randomized trials are needed. Treatment of mild and moderate glaucoma can differ; however, simple therapies should always be re-evaluated before starting any kind of invasive surgery.

Furthermore, MIGS is often combined with cataract surgery showing potentially a greater IOP reduction than stand-alone procedures. In this study, only stand-alone surgery was analyzed. The effect of combined interventions should be evaluated in a subgroup analysis.

Nevertheless, to the best of our knowledge, this is the first independent study comparing the outcome of iStent inject ${ }^{\circledR}$ and Trabectome ${ }^{\circledR}$ surgery with a selective laser procedure.

\section{CONCLUSION}

Our results show that iStent inject ${ }^{\circledR}$ and Trabectome $^{\circledR}$ surgery are safe and effective surgeries and-regarding the IOP reduction and complication profile-are comparable to the outcome after SLT intervention. A gradual therapeutic scheme can be recommended with SLT as an initial procedure as it changes the anatomic structure of the iridocorneal angle the least. Patient selection in considering a wellpigmented trabecular meshwork and an open angle is fundamental for success of selective laser treatment. If the IOP is not sufficiently lowered, second-line Trabectome ${ }^{\circledR}$ and iStent inject $^{\circledR}$ surgery is still possible. Klamann et al. and Maier et al. have already shown that prior SLT treatment does not influence the outcome following Trabectome ${ }^{\circledR}$ and iStent inject ${ }^{\circledR}$ surgery in open-angle glaucoma $[13,50]$.

\section{ACKNOWLEDGEMENTS}

We sincerely thank the participants of this study. 
Funding. This research did not receive any specific grant from funding agencies in the public, commercial, or not-for-profit sectors. None of the authors have a financial interest. No funding or sponsorship was received for this study. For publication the Rapid Service Fee was partly funded by the authors and Charité Universitätsmedizin Berlin.

Authorship. All named authors meet the International Committee of Medical Journal Editors (ICMJE) criteria for authorship for this article, take responsibility for the integrity of the work as a whole, and have given their approval for this version to be published.

Author's Contributions. Milena Pahlitzsch concept and design, statistical analysis, writing, drafting the manuscript. Anja-Maria Davids concept and design, writing, drafting the manuscript. Sibylle Winterhalter - statistical analysis, revising the manuscript. Malte Zorn data collection, statistical analysis. Emanuel Reitemeyer - data collection, writing. Matthias KJ Klamann - data collection, writing, figure creation. Necip Torun - data collection, writing, surgery. Eckart Bertelmann - data collection, writing, surgery. Anna-Karina Maier concept, writing, revising the manuscript.

Disclosures. Milena Pahlitzsch, Anja-Maria Davids, Necip Torun, Malte Zorn, Emanuel Reitemayer, Anna-Karina Maier, and Matthias Klamann certify that they have no affiliations with or involvement in any organization or entity with any financial interest (such as honoraria; educational grants; participation in speakers' bureaus; membership, employment, consultancies, stock ownership, or other equity interest; and expert testimony or patent-licensing arrangements), or non-financial interest (such as personal or professional relationships, affiliations, knowledge, or beliefs). The authors have made the following disclosures: Sibylle Winterhalter: Consultant-Allergan, Novartis, Bayer, MSD, Heidelberg Engineering. Eckart Bertelmann: Consultant-Hoya, Zeiss, Human Optics, Alcon.
Compliance with Ethics Guidelines. Ethics approval had been given by the Ethikkommission, Charité - Universitätsmedizin Berlin, EA4/ $047 / 20$. For this type of study formal consent is not required. This study adhered to the ethical standards of the Declaration of Helsinki of 1964 and its later amendments. Informed written consent was obtained from all individual participants prior to surgery or laser treatment included in the study.

Data Availability. The datasets generated during and/or analyzed during the current study are available from the corresponding author on reasonable request (following Charité - Universitätsmedizin Berlin guidelines for retrospective research).

Open Access. This article is licensed under a Creative Commons Attribution-NonCommercial 4.0 International License, which permits any non-commercial use, sharing, adaptation, distribution and reproduction in any medium or format, as long as you give appropriate credit to the original author(s) and the source, provide a link to the Creative Commons licence, and indicate if changes were made. The images or other third party material in this article are included in the article's Creative Commons licence, unless indicated otherwise in a credit line to the material. If material is not included in the article's Creative Commons licence and your intended use is not permitted by statutory regulation or exceeds the permitted use, you will need to obtain permission directly from the copyright holder. To view a copy of this licence, visit http://creativecommons.org/licenses/by$\mathrm{nc} / 4.0 /$.

\section{REFERENCES}

1. Lavia C, Dallorto L, Maule M, Ceccarelli M, Fea AM. Minimally-invasive glaucoma surgeries (MIGS) for open angle glaucoma: a systematic review and meta-analysis. PLoS ONE. 2017;12(8):e0183142.

2. Pillunat LE, Erb C, Junemann AG, Kimmich F. Micro-invasive glaucoma surgery (MIGS): a review of surgical procedures using stents. Clin Ophthalmol. 2017;11:1583-600. 
3. Agrawal P, Bradshaw SE. Systematic literature review of clinical and economic outcomes of microinvasive glaucoma surgery (MIGS) in primary openangle glaucoma. Ophthalmol Ther. 2018;7(1): 49-73.

4. Chow JTY, Hutnik CML, Solo K, Malvankar-Mehta MS. When is evidence enough evidence? A systematic review and meta-analysis of the trabectome as a solo procedure in patients with primary openangle glaucoma. J Ophthalmol. 2017:2965725. https://doi.org/10.1155/2017/2965725.

5. Klamann MK, Gonnermann J, Pahlitzsch M, et al. iStent inject in phakic open angle glaucoma. Graefes Arch Clin Exp Ophthalmol. 2015;253(6):941-7.

6. Minckler D, Mosaed S, Dustin L, Ms BF. Trabectome (trabeculectomy-internal approach): additional experience and extended follow-up. Trans Am Ophthalmol Soc. 2008;106:149-59 (discussion 159-160).

7. Minckler DS, Baerveldt G, Alfaro MR, Francis BA. Clinical results with the Trabectome for treatment of open-angle glaucoma. Ophthalmology. 2005;112(6):962-7.

8. Voskanyan L, García-Feijoó J, Belda JI, et al. Prospective, unmasked evaluation of the iStent ${ }^{\circledR}$ inject system for open-angle glaucoma: synergy trial. Adv Ther. 2014;31(2):189-201.

9. Garg A, Gazzard G. Selective laser trabeculoplasty: past, present, and future. Eye. 2018;32(5):863-76.

10. Latina MA, Park C. Selective targeting of trabecular meshwork cells: in vitro studies of pulsed and CW laser interactions. Exp Eye Res. 1995;60(4):359-71.

11. Jonas JB. Biomorphometrie des Nervus Opticus. Stuttgart, Germany: Enke; 1989. pp 1-148.

12. Goldmann H, Schmidt T. Applanation tonometry. Ophthalmologica. 1957;134(4):221-42.

13. Klamann MK, Gonnermann J, Maier AK, Bertelmann E, Joussen AM, Torun N. Influence of selective laser trabeculoplasty (SLT) on combined clear cornea phacoemulsification and trabectome outcomes. Graefes Arch Clin Exp Ophthalmol. 2014;252(4):627-31.

14. Avery N, Ang GS, Nicholas S, Wells A. Repeatability of primary selective laser trabeculoplasty in patients with primary open-angle glaucoma. Int Ophthalmol. 2013;33:501-6.

15. Hong BK, Winer JC, Martone JF, Wand M, Altman B, Shields B. Repeat selective laser trabeculoplasty. J Glaucoma. 2009;18:180-3.
16. Jordan JF, Wecker $\mathrm{T}$, van Oterendorp $\mathrm{C}$, et al. Trabectome surgery for primary and secondary open angle glaucomas. Graefes Arch Clin Exp Ophthalmol. 2013;251(12):2753-60.

17. Ting JL, Damji KF, Stiles MC. Ab interno trabeculectomy: outcomes in exfoliation versus primary open-angle glaucoma. J Cataract Refract Surg. 2012;38(2):315-23.

18. Pahlitzsch M, Gonnermann J, Maier AK, et al. Trabeculectomy Ab interno in primary open angle glaucoma and exfoliative glaucoma. Klin Monbl Augenheilkd. 2015;232(10):1198-207.

19. Mizoguchi T, Nishigaki S, Sato T, Wakiyama $H$, Ogino N. Clinical results of Trabectome surgery for open-angle glaucoma. Clin Ophthalmol. 2015;9: 1889-94.

20. Fea AM, Belda JI, Rekas M, et al. Prospective unmasked randomized evaluation of the iStent inject $^{\circledR}$ versus two ocular hypotensive agents in patients with primary open-angle glaucoma. Clin Ophthalmol. 2014;8:875-82.

21. Ahmed II, Katz LJ, Chang DF, et al. Prospective evaluation of microinvasive glaucoma surgery with trabecular microbypass stents and prostaglandin in open-angle glaucoma. J Cataract Refract Surg. 2014;40(8):1295-300.

22. Lindstrom R, Lewis R, Hornbeak DM, et al. Outcomes following implantation of two second-generation trabecular micro-bypass stents in patients with open-angle glaucoma on one medication: 18-month follow-up. Adv Ther. 2016;33(11): 2082-90.

23. Donnenfeld ED, Solomon KD, Voskanyan L, et al. A prospective 3-year follow-up trial of implantation of two trabecular microbypass stents in open-angle glaucoma. Clin Ophthalmol. 2015;9:2057-65.

24. Katz LJ, Erb C, Carceller GA, et al. Prospective, randomized study of one, two, or three trabecular bypass stents in open-angle glaucoma subjects on topical hypotensive medication. Clin Ophthalmol. 2015;9:2313-20.

25. Vold SD, Voskanyan L, Tetz M, et al. Newly diagnosed primary open-angle glaucoma randomized to 2 trabecular bypass stents or prostaglandin: outcomes through 36 months. Ophthalmol Ther. 2016;5(2):161-72.

26. Pahlitzsch M, Torun N, Gonnermann J, et al. Trabeculectomy ab interno (trabectome): yet another possibility in the treatment of uncontrolled glaucomatocyclitic crisis under systemic valganciclovir therapy? Eye. 2015;29(10):1335-9. 
27. Pahlitzsch M, Gonnermann J, Maier AK, Bertelmann E, Klamann MK, Erb C. Modified goniotomy as an alternative to trabectome in primary open angle glaucoma and pseudoexfoliation glaucoma: 1 year results. Can J Ophthalmol. 2017;52(1):92-8.

28. Maeda M, Watanabe M, Ichikawa K. Evaluation of trabectome in open-angle glaucoma. J Glaucoma. 2013;22(3):205-8.

29. Jea SY, Francis BA, Vakili G, Filippopoulos T, Rhee $\mathrm{DJ}$. Ab interno trabeculectomy versus trabeculectomy for open-angle glaucoma. Ophthalmology. 2012;119(1):36-42.

30. Rich JT, Neely JG, Paniello RC, Voelker CC, Nussenbaum B, Wang EW. A practical guide to understanding Kaplan-Meier curves. Otolaryngol Head Neck Surg. 2010;143(3):331-6.

31. Qian SH, Sun XH. Selective laser trabeculoplasty in the treatment of primary open-angle glaucoma. Zhonghua Yi Xue Za Zhi. 2007;87(2):118-20.

32. Werner M, Smith MF, Doyle JW. Selective laser trabeculoplasty in phakic and pseudophakic eyes. Ophthalmic Surg Lasers Imaging. 2007;38(3):182-8.

33. Babighian S, Caretti L, Tavolato M, Cian R, Galan A. Excimer laser trabeculotomy vs 180 degrees selective laser trabeculoplasty in primary open-angle glaucoma. A 2-year randomized, controlled trial. Eye. 2010;24(4):632-8.

34. Shazly TA, Smith J, Latina MA. Long-term safety and efficacy of selective laser trabeculoplasty as primary therapy for the treatment of pseudoexfoliation glaucoma compared with primary open-angle glaucoma. Clin Ophthalmol. 2010;5:5-10.

35. Juzych MS, Chopra V, Banitt MR, et al. Comparison of long-term outcomes of selective laser trabeculoplasty versus argon laser trabeculoplasty in openangle glaucoma. Ophthalmology. 2004;111(10): 1853-9.

36. Weinand FS, Althen F. Long-term clinical results of selective laser trabeculoplasty in the treatment of primary open angle glaucoma. Eur J Ophthalmol. 2006;16(1):100-4.

37. Rosenfeld E, Shemesh G, Kurtz S. The efficacy of selective laser trabeculoplasty versus argon laser trabeculoplasty in pseudophakic glaucoma patients. Clin Ophthalmol. 2012;6:1935-40.

38. Katz LJ, Steinmann WC, Kabir A, Molineaux J, Wizov SS, Marcellino G. Selective laser trabeculoplasty versus medical therapy as initial treatment of glaucoma: a prospective, randomized trial. J Glaucoma. 2012;21(7):460-8.
39. Liu Y, Birt CM. Argon versus selective laser trabeculoplasty in younger patients: 2-year results. J Glaucoma. 2012;21(2):112-5.

40. Bovell AM, Damji KF, Hodge WG, Rock WJ, Buhrmann RR, Pan YI. Long term effects on the lowering of intraocular pressure: selective laser or argon laser trabeculoplasty? Can J Ophthalmol. 2011;46(5): 408-13.

41. Lai JS, Chua JK, Tham CC, Lam DS. Five-year follow up of selective laser trabeculoplasty in Chinese eyes. Clin Exp Ophthalmol. 2004;32(4):368-72.

42. Leahy KE, White AJ. Selective laser trabeculoplasty: current perspectives. Clin Ophthalmol. 2015;9: 833-41.

43. Gonnermann J, Bertelmann E, Pahlitzsch M, MaierWenzel AB, Torun N, Klamann MK. Contralateral eye comparison study in MICS \& MIGS: Trabectome ${ }^{\circledR}$ vs. iStent inject ${ }^{\circledR}$. Graefes Arch Clin Exp Ophthalmol. 2017;255(2):359-65.

44. Kurji K, Rudnisky CJ, Rayat JS, et al. Phaco-trabectome versus phaco-iStent in patients with openangle glaucoma. Can J Ophthalmol. 2017;52(1): 99-106.

45. Khan M, Saheb H, Neelakantan A, et al. Efficacy and safety of combined cataract surgery with 2 trabecular microbypass stents versus ab interno trabeculotomy. J Cataract Refract Surg. 2015;41(8): 1716-24.

46. Berdahl JP, Khatana AK, Katz LJ, et al. Cost-comparison of two trabecular micro-bypass stents versus selective laser trabeculoplasty or medications only for intraocular pressure control for patients with open-angle glaucoma. J Med Econ. 2017;20(7): 760-6.

47. De Keyser M, De Belder M, De Belder J, De Groot V. Selective laser trabeculoplasty as replacement therapy in medically controlled glaucoma patients. Acta Ophthalmol. 2017;96(5):e577-81.

48. Kennedy JB, SooHoo JR, Kahook MY, Seibold LK. Selective laser trabeculoplasty: an update. Asia Pac J Ophthalmol. 2016;5(1):63-9.

49. Song J. Complications of selective laser trabeculoplasty: a review. Clin Ophthalmol. 2016;10:137-43.

50. Maier AB, Arani P, Pahlitzsch M, et al. Influence of selective laser trabeculoplasty (SLT) on the iStent inject ${ }^{\circledR}$ outcomes. BMC Ophthalmol. 2020;20(1): 457. 